Research Paper

\title{
Lower serum bilirubin is associated with poor renal outcome in IgA nephropathy patients
}

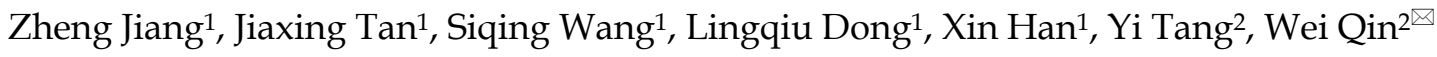 \\ 1. West China School of Medicine, Sichuan University \\ 2. Division of Nephrology, Department of Medicine, West China Hospital of Sichuan University \\ $\square$ Corresponding author: Wei Qin , MD, PhD, Division of Nephrology, Department of Medicine, West China Hospital of Sichuan University, Chengdu 610041, \\ Sichuan. Address: No.37, Guoxue Alley, Chengdu, Sichuan Province, China. Email: qinweihx@scu.edu.cn \\ (C) The author(s). This is an open access article distributed under the terms of the Creative Commons Attribution License (https://creativecommons.org/licenses/by/4.0/). \\ See http://ivyspring.com/terms for full terms and conditions.
}

Received: 2021.03.04; Accepted: 2021.05.19; Published: 2021.06.11

\begin{abstract}
Aims: $\lg$ A nephropathy ( $\lg A N)$ is the most prevalent primary glomerulonephritis worldwide. We conducted this study to explore the relationship between serum bilirubin and renal outcome of patients with IgAN.

Methods: A total of 1492 biopsy proven IgAN patients were recruited and divided into two groups according to their median serum bilirubin concentration: the low bilirubin group (serum bilirubin $\leq 9.7 \mathrm{umol} / \mathrm{L}, \mathrm{n}=753$ ) and high bilirubin group (serum bilirubin>9.7umol/L, $n=739$ ). Basic clinical characteristics were assessed at the time of renal biopsy and the relationships between serum bilirubin and the combined endpoints were analyzed. The combined endpoints were defined as a $50 \%$ decline in estimate glomerular filtration rate (e-GFR), end-stage kidney disease (ESKD), renal transplantation and/or death. In addition, propensity score matching (PSM) was then performed to improve balance and simulate randomization between patients in different groups. Kaplan-Meier survival analysis was applied to explore the role of serum bilirubin in the progression of IgAN. Three clinicopathological models of multivariate Cox regression analysis were established to evaluate the association of serum bilirubin and renal prognosis of IgAN.

Results: During median 5-year follow-up period, significant differences were shown in Kaplan-Meier analysis. In the unmatched group, 189 (12.7\%) patients progressed to the renal combined endpoints.

Among this, 122 in 753 patients (16.2\%) were in low bilirubin group and 67 in 739 patients (9.1\%) were in high bilirubin group $(p<0.001)$. After PSM, there were 134 (11.8\%) patients reached the combined endpoints, which included 77 in 566 patients (14.6\%) in low bilirubin group and 57 in 566 patients (10.1\%) in high bilirubin group $(p=0.039)$. The results of three models (including demographics, pathological, clinical indicators and serum bilirubin) demonstrated that a lower basic serum bilirubin level was significantly associated with a higher risk of reaching combined endpoints in IgAN patients both in unmatched and matched cohort.
\end{abstract}

Conclusion: Serum bilirubin level may be negatively associated with the progression of IgAN.

Key words: end-stage kidney disease, IgA nephropathy, serum bilirubin, propensity score matching.

\section{Introduction}

Immunoglobin A nephropathy (IgAN) is featured by IgA diffusely depositing in the kidneys, which is the most prevalent primary glomerulonephritis worldwide. Nearly $15-40 \%$ of IgAN patients will progress to end stage renal disease (ESRD) within 20 years [1] .It is quite meaningful to identify risk factors related to the progression of IgAN. Early intervention of these factors may delay the development of IgAN. It is reported that the pathogenesis of IgAN is associated with oxidative stress [2-4]. The dynamic balance between oxidation and antioxidation has been destroyed in patients with IgAN [4]. It was reported that intrarenal immunoreactivity of heme oxygenase- $1(\mathrm{HO}-1)$ was increased in $\operatorname{IgAN}$ [5], which can degrade heme to produce carbon monoxide, iron and biliverdin (BV), which is reduced to bilirubin by biliverdin reductase [6]. Therefore, serum bilirubin is regarded as a powerful endogenous antioxidant. Many studies have clarified the role of serum bilirubin in diabetes mellitus, renal transplantation, stroke, cardiovascular and metabolic disease [7-9]. However, there still lack 
of research on the relationship between serum bilirubin and IgAN. Whether the level of serum bilirubin could be another predictor of progression of IgAN remains unknown. To clarify these issues, we conducted this study.

\section{Materials and Methods}

\section{Patients}

Biopsy-proven IgAN patients from West China hospital of Sichuan university between May 2009 and September 2019 were enrolled in this study. The Inclusion criteria were: (1) patients confirmed as IgAN by renal biopsy; (2) patients were followed up for at least 6 months or reached the endpoint of study; (3) patients $\geq 14$ years old. The exclusion criteria applied were as follow: (1) patients with systemic disease, such as systemic lupus erythematosus (SLE), Henoch-Schönlein purpura (HSP), liver cirrhosis or disorder of liver function, malignancy etc.; (2) patients without complete renal biopsy or clinical data. Patients were followed up in the out-patient department of West China Hospital or by regular phone calls. The study was approved by the Ethical Committee of West China Hospital of Sichuan University. Informed consent was obtained from each patient or legal guardians prior to treatment.

\section{Data Collection}

Electronic medical records were used to obtain patients' information including age, gender, clinical manifestations, laboratory indexes, renal pathology report, systolic and diastolic blood pressure and treatment strategies. Laboratory values included $24 \mathrm{~h}$ proteinuria (UPRO), hematuria level (URBC), hemoglobin $(\mathrm{Hb})$, serum total bilirubin $(\mathrm{TB})$, conjugated bilirubin (CB), unconjugated bilirubin (UCB), alanine aminotransferase (ALT), aspartate aminotransferase (AST), serum albumin (ALB), serum creatinine (Scr), estimate glomerular filtration rate (eGFR), uric acid (UA), triglycerides (TG), total cholesterol (TC). The Chronic Kidney Disease Epidemiology Collaboration (CKD-EPI) formula was used to calculate eGFR. Nephrotic syndrome (NS) was defined as $24 \mathrm{~h}$ proteinuria $>3.5 \mathrm{~g} / \mathrm{d}$ and serum albumin $<30 \mathrm{~g} / \mathrm{L}$. Anemia was defined as hemoglobin $<110 \mathrm{~g} / \mathrm{L}$ in women and $<120 \mathrm{~g} / \mathrm{L}$ in men. The pathological definitions of mesangial proliferation, endocapillary proliferation, segmental glomerulosclerosis, tubular atrophy/interstitial fibrosis, crescents were according to the Oxford classification [10].

\section{Treatments}

Treatment regimens were decided by patients and their doctors according to the clinical and pathological features of the patients. All patients received optimal support treatment including full dose of ACEI/ARB. Glucocorticoids were used when there was persistent proteinuria $(>1 \mathrm{~g} / \mathrm{d})$ after optimal support treatment, $0.5-1 \mathrm{mg} / \mathrm{kg}$ prednisone daily and tapering down gradually within 6-8 months. Immunosuppressants would be considered in patients with impaired kidney function $(\mathrm{Scr}>1.5$ $\mathrm{mg} / \mathrm{dl}$ ) or rapidly progressive kidney function decline. Immunosuppressants included cyclophosphamide $(2 \mathrm{mg} / \mathrm{kg}$ daily for 3 months), mycophenolate mofetil (1-2g daily for 6-8 months), tacrolimus $(0.03-0.05 \mathrm{mg} / \mathrm{kg}$ daily for $6-8$ months) or cyclosporin (3-5 mg/ $\mathrm{kg}$ daily for 6-8 months).

\section{Outcomes}

The combined endpoints of renal outcome were eGFR decreased $\geq 50 \%$ of the baseline level, end-stage kidney disease (ESKD), renal transplantation and/or death. ESKD was defined as eGFR $\leq 15 \mathrm{~mL} / \mathrm{min} / 1.73$ $\mathrm{m}^{2}$ or maintenance renal replacement treatment.

\section{Statistical analyses}

Participants were divided into two groups according to their median baseline serum bilirubin concentration: the low-bilirubin group (serum bilirubin $\leq 9.7 \mathrm{umol} / \mathrm{L}, \quad \mathrm{n}=753$ ) and high-bilirubin group (serum bilirubin $>9.7 \mathrm{umol} / \mathrm{L}, \mathrm{n}=739$ ). To lessen the selection bias between different groups in this study, propensity score matching (PSM) was performed to adjust for sex, ALT, TG, TC, T, C, eGFR at a 1:1 ratio. PSM was conducted by "Match It" $\mathrm{R}$ package and the "nearest neighbor matching" method. A caliper of 0.18 propensity score standard deviation was used to match. Two-tailed $\mathrm{p}<0.05$ was considered statistically significant. Categorical data were described as percentages and analyzed using the Chi-square tests. Continuous variables were expressed as mean \pm SD or median (first-third interquartile range) and analyzed with the ANOVA, Student's $\mathrm{t}$ test or nonparametric Mann-Whitney $\mathrm{U}$ test. Kaplan-Meier survival analysis was performed using the log-rank test. The effects of clinical manifestations and pathological features on renal outcomes were assessed by mutivariate Cox regression analysis. All statistical analysis was performed by using IBM SPSS statistics 26.0 software.

\section{Results}

\section{Baseline characteristics}

1617 IgAN patients were recruited in our study. Among this cohort, 125 patients were excluded because of liver dysfunction or cirrhosis (24), diabetes with uncontrolled blood glucose (47) and lack of renal biopsy information or clinical data (54). Finally, 1492 
patients were enrolled in this study. The median serum bilirubin of participates was 9.7umol/L (range 2.4-29.7umol/L). And the patients were divided into two groups according to median serum bilirubin concentration: low bilirubin group (LB group: TB $\leq 9.7 \mathrm{umol} / \mathrm{L}, \mathrm{n}=753$ ) and high bilirubin group (HB group: TB $>9.7$ umol/L, n=739) (Figure 1). Patients in LB group had a lower proportion of male $(37.5 \%$, $\mathrm{p}<0.001)$, lower glucocorticoids (GC) treatment $(69.5 \%, \mathrm{p}=0.002)$, higher nephrotic syndrome $(12.2 \%$, $\mathrm{p}<0.001)$, severer anemia $(16.9 \%, \mathrm{p}<0.001)$, renal failure $(27.9 \%, \mathrm{p}<0.001)$ and pathological lesions. While patients in HB group tended to have a higher level of $\mathrm{UCB}(\mathrm{p}<0.001), \mathrm{CB}(\mathrm{p}<0.001)$, ALT $(\mathrm{p}=0.001)$ and lower TG $(p<0.001), \mathrm{TC}(\mathrm{p}<0.001)$ level. In order to eliminate the difference between two groups, 1132 patients (566 in each group) were enrolled after propensity score matching at a 1:1 ratio. Then there were no differences in clinicopathological manifestations and treatments except serum conjugated and unconjugated bilirubin levels (Table $1)$.

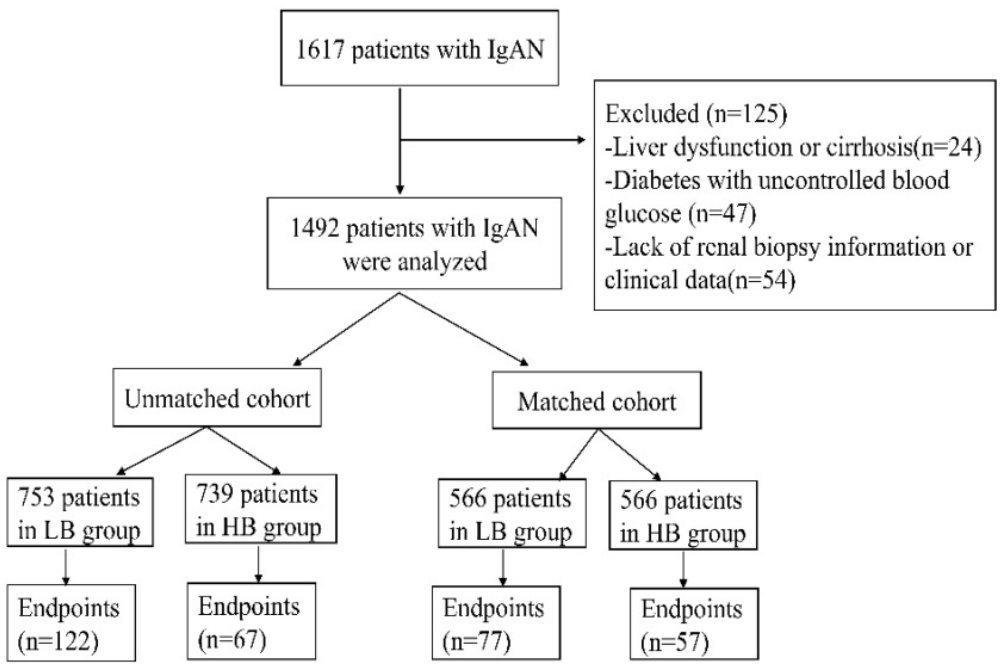

Figure 1: Flow chart of the study.

Table 1: Baseline characteristics of $\lg A N$ patients.

\begin{tabular}{|c|c|c|c|c|c|c|}
\hline \multirow[t]{2}{*}{ Variables } & \multicolumn{2}{|c|}{ Before PSM serum bilirubin groups } & \multirow[b]{2}{*}{$P$ value } & \multicolumn{2}{|c|}{ After PSM serum bilirubin groups } & \multirow[b]{2}{*}{$P$ value } \\
\hline & $\mathrm{LB}(\mathrm{n}=753) \leq 9.7 \mathrm{mmol} / \mathrm{L}$ & $\mathrm{HB}(\mathrm{n}=739)>9.7 \mathrm{mmo} / \mathrm{L}$ & & $\mathrm{LB}(\mathrm{n}=566) \leq 9.7 \mathrm{mmol} / \mathrm{L}$ & $\mathrm{HB}(\mathrm{n}=566)>9.7 \mathrm{mmo} / \mathrm{L}$ & \\
\hline $\operatorname{Sex}(\mathrm{F} / \mathrm{M}, \%)$ & $471 / 282(62.5 / 37.5)$ & $350 / 389(47.4 / 52.6)$ & $<0.001 * *$ & $338 / 228(59.7 / 40.3)$ & $332 / 234(58.7 / 41.3)$ & 0.762 \\
\hline Age(year) & $33(25-42)$ & $32(26-40)$ & 0.135 & $34.49 \pm 11.26$ & $34.45 \pm 10.49$ & 0.952 \\
\hline Follow-up (m) & $58.55 \pm 27.74$ & $61.26 \pm 28.08$ & 0.061 & $59.64 \pm 27.77$ & $62.07 \pm 27.73$ & 0.141 \\
\hline ALT (IU/L) & $18(13-25)$ & $19(14-30)$ & $0.001^{\text {** }}$ & $18(13-26)$ & $18(13-29)$ & 0.058 \\
\hline AST (IU/L) & $22.34 \pm 8.64$ & $22.88 \pm 8.32$ & 0.219 & $22.16 \pm 8.03$ & $22.69 \pm 8.5$ & 0.284 \\
\hline $\mathrm{TG}(\mathrm{mmol} / \mathrm{L})$ & $1.62(1.08-2.4)$ & $1.4(0.99-1.94)$ & $<0.001^{* *}$ & $1.82 \pm 1.21$ & $1.82 \pm 1.34$ & 0.951 \\
\hline $\mathrm{TC}(\mathrm{mmol} / \mathrm{L})$ & $4.93(4.17-5.99)$ & $4.7(3.94-5.42)$ & $<0.001^{* *}$ & $4.93 \pm 1.28$ & $5 \pm 1.31$ & 0.376 \\
\hline URBC (/HP) & $22(7-71.5)$ & $17(6-52)$ & 0.091 & $20(7-65.25)$ & $16(6-51)$ & 0.055 \\
\hline UA (umol/L) & $373.48 \pm 107.84$ & $377.01 \pm 100.35$ & 0.512 & $370.38 \pm 107.55$ & $373.4 \pm 102.33$ & 0.629 \\
\hline $\mathrm{LDH}(\mathrm{IU} / \mathrm{L})$ & $179.28 \pm 47.93$ & $176.62 \pm 42.56$ & 0.268 & $173.63 \pm 41.2$ & $179.03 \pm 51.75$ & 0.056 \\
\hline UCB(umol/L) & $5.1(4.1-6.1)$ & $9.4(7.9-11.6)$ & $<0.001^{* *}$ & $5.1(4.2-6.1)$ & $9.5(8-11.6)$ & $<0.001^{* *}$ \\
\hline CB (umol/L) & $2(1.5-2.6)$ & $4(3.2-5.1)$ & $<0.001^{* *}$ & $2.2(1.7-2.73)$ & $3.85(3-4.8)$ & $<0.001^{* *}$ \\
\hline $\operatorname{ALB}(\mathrm{g} / \mathrm{L})$ & $38.3(33.9-42.1)$ & $41.2(38.3-44.3)$ & $<0.001^{* *}$ & $39.63 \pm 5.62$ & $39.58 \pm 5.51$ & 0.865 \\
\hline $\operatorname{UPRO}(\mathrm{g} / \mathrm{d})$ & $1.89(0.98-3.16)$ & $1.16(0.69-2.09)$ & $<0.001^{* *}$ & $2.06 \pm 2.39$ & $1.84 \pm 1.8$ & 0.078 \\
\hline NS (N/Y, \%) & $661 / 92(87.8 / 12.2)$ & $724 / 15(98 / 2)$ & $<0.001^{* *}$ & $551 / 15(97.3 / 22.7)$ & $551 / 15(97.3 / 22.7)$ & 1 \\
\hline HTN (N/Y, \%) & $549 / 204(72.9 / 27.1)$ & $546 / 193(73.9 / 26.1)$ & 0.682 & $422 / 144(74.6 / 25.4)$ & $418 / 148(73.9 / 26.1)$ & 0.839 \\
\hline Anemia (N/Y, \%) & $626 / 127(83.1 / 16.9)$ & $690 / 49(93.4 / 6.6)$ & $<0.001^{* *}$ & $512 / 54(90.5 / 9.5)$ & $517 / 49(91.3 / 8.7)$ & 0.679 \\
\hline eGFR (\%) & & & $<0.001^{* *}$ & & & 0.607 \\
\hline$<60 \mathrm{ml} / \mathrm{min}$ & $210(27.9)$ & $120(16.2)$ & & $121(21.4)$ & $113(20)$ & \\
\hline$>60 \mathrm{ml} / \mathrm{min}$ & $543(72.1)$ & $619(83.8)$ & & 445 (78.6) & $453(80)$ & \\
\hline M0/M1(\%) & $169 / 584(22.4 / 77.6)$ & $196 / 543(26.5 / 73.5)$ & 0.071 & $133 / 433(23.5 / 76.5)$ & $144 / 422(25.4 / 74.6)$ & 0.489 \\
\hline E0/E1(\%) & $708 / 45(94 / 6)$ & $716 / 23(96.9 / 3.1)$ & $0.009^{*}$ & $539 / 27(95.2 / 4.8)$ & $546 / 20(96.5 / 3.5)$ & 0.372 \\
\hline S0/S1(\%) & $307 / 446(40.8 / 59.2)$ & $307 / 432(41.5 / 58.5)$ & 0.793 & $230 / 336(40.6 / 59.4)$ & $219 / 347(38.7 / 61.3)$ & 0.544 \\
\hline T0/T1(\%) & $559 / 194(74.2 / 25.8)$ & $628 / 111(85 / 15)$ & $<0.001^{* *}$ & $449 / 117$ (79.3/20.7) & $460 / 106(81.3 / 18.7)$ & 0.455 \\
\hline $\mathrm{C} 0 / \mathrm{C} 1(\%)$ & $567 / 186(75.3 / 24.7)$ & $593 / 146(80.2 / 19.8)$ & $0.025^{*}$ & $443 / 123(78.3 / 21.7)$ & $443 / 123(78.3 / 21.7)$ & 1 \\
\hline GC (N/Y) & $230 / 523(30.5 / 69.5)$ & $174 / 565(23.5 / 76.5)$ & $0.002^{*}$ & $159 / 407(28.1 / 71.9)$ & $141 / 425(24.9 / 75.1)$ & 0.252 \\
\hline
\end{tabular}


A.

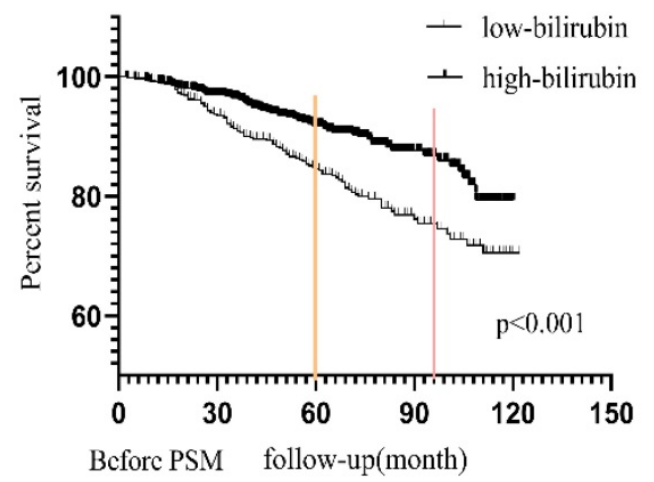

B.

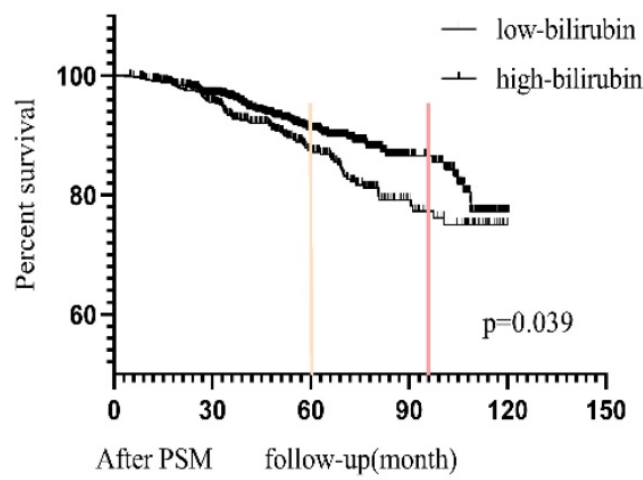

$\begin{array}{lllll}566 & 502 & 237 & 70 & 24\end{array}$ low-bilirubin $\begin{array}{lllll}753 & 655 & 309 & 86 & 28\end{array}$

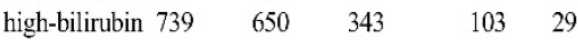

$\begin{array}{lllll}566 & 501 & 267 & 82 & 21\end{array}$

Figure 2: Probability of renal survival between high bilirubin group and low bilirubin group in the unmatched and matched cohort.

\section{Long term renal survival}

The Kaplan-Meier survival curves of the unmatched cohort and matched populations according to the level of serum bilirubin were shown in Figure 2(A and B). During median 5-year follow-up period, in the unmatched cohort, 189 (12.7\%) patients progressed to the renal endpoints. It could be noticed that more patients in LB group (122 in 753 patients, $16.2 \%)$ reached renal endpoints than those in $\mathrm{HB}$ group (67 in 739 patients, 9.1\%). In the matched cohort, there were $134(11.8 \%)$ patients reached the combined endpoints, which included 77 in 566 patients (14.6\%) in LB group and 57 in 566 patients $(10.1 \%)$ in HB group. Kaplan-Meier analysis indicated that better renal survival in high bilirubin group compared with those in low bilirubin group in unmatched and matched cohort $(\mathrm{p}<0.001$ and $\mathrm{p}=0.039$, Figure 2). It was found that 5-year renal survival rates were $84.8 \%(87.5 \%$ after PSM) and 91.9\% (91\% after PSM) in LB and HB groups. 8-year renal survival rates were $75.2 \%$ (77.3\% after PSM) and $86.9 \%$ (86.5\% after PSM) in two groups, respectively.

\section{Factors of renal endpoints}

Renal endpoints included eGFR decreased $\geq 50 \%$ of the baseline level, end-stage kidney disease (ESKD), renal transplantation and/or death. Multivariate Cox regression model was performed to analysis the relationship between clinicopathological parameters and renal endpoint (Table 2-4). In the unmatched cohort, model 1 (demographics + pathological features + serum total bilirubin), model 2 (demographics + clinical features + serum total bilirubin) and model 3 (demographics + clinical+ pathological features+ serum total bilirubin) indicated that serum bilirubin was independent factors of renal endpoints (model 1:HR 0.646, 95\%CI 0.476-0.877,
$\mathrm{P}=0.005$; model 2: HR 0.624 ，95\%CI $0.451-0.865$, $\mathrm{P}=0.005$; model 3:HR 0.651, 95\%CI 0.467-0.906, $\mathrm{P}=0.011)$. After PSM, the results of model 1(HR 0.705, 95\% CI 0.5-0.994, $\mathrm{P}=0.046$ ), model 2(HR 0.57, 95\%CI $0.317-0.819, \mathrm{P}=0.002$ ) and model $3(\mathrm{HR} 0.568,95 \% \mathrm{CI}$ $0.394-0.819, \mathrm{P}=0.002$ ) also strongly indicated serum bilirubin as a protective factor of renal prognosis. Based on these results, it could be confirmed that serum bilirubin was an independent protective factor of renal outcomes in IgAN patients.

Table 2: Analysis of factors associated with renal outcomes in model 1 (demographics + pathological features+ serum bilirubin).

\begin{tabular}{lllllll}
\hline Variables & \multicolumn{3}{l}{ Before PSM } & \multicolumn{4}{l}{ After PSM } \\
\cline { 2 - 7 } & HR & $95 \%$ CI & P & HR & $95 \%$ CI & P \\
\hline Male/female & 1.427 & $1.064-1.914$ & $0.018^{*}$ & 1.573 & $1.102-2.245$ & $0.013^{*}$ \\
Age (per year) & 0.998 & $0.984-1.012$ & 0.757 & 1.004 & $0.988-1.021$ & 0.61 \\
Total bilirubin (High/low) & 0.646 & $0.476-0.877$ & $0.005^{*}$ & 0.705 & $0.5-0.994$ & $0.046^{*}$ \\
M(M1/M0) & 1.321 & $0.737-2.369$ & 0.35 & 1.156 & $0.694-1.925$ & 0.578 \\
E(E1/E0) & 1.373 & $0.765-2.464$ & 0.289 & 1.245 & $0.548-2.828$ & 0.601 \\
S(S1/S0) & 1.877 & $1.319-2.67$ & $<0.001^{* *}$ & 2.198 & $1.408-3.429$ & $0.001^{* *}$ \\
T(T1-2/T0) & 5.995 & $4.36-8.244$ & $<0.001^{* *}$ & 5.602 & $3.834-8.186$ & $<0.001^{* *}$ \\
C(C1-2/C0) & 1.008 & $0.722-1.407$ & 0.964 & 0.876 & $0.57-1.346$ & 0.547 \\
\hline
\end{tabular}

Notes: M mesangial hypercellularity, E endocapillary hypercellularity, S segmental glomerulosclerosis or adhesion, $\mathrm{T}$ tubular atrophy/interstitial fibrosis, $\mathrm{C}$ crescents, ${ }^{*}: \mathrm{p}<0.05,{ }^{* *}: \mathrm{p} \leq 0.001$.

\section{Discussion}

Although the detailed mechanism of IgAN still remains unknown, the role of oxidative stress in the pathogenesis has attracted more and more attention $[2,11,12]$. It was reported that the activation of intracellular signaling in mesangial cells cultured with aberrantly glycosylated IgA and in renal biopsies of IgAN patients basically results in oxidative stress [5]. Advanced oxidation protein products (AOPPs) are considered to be important mediators of inflammation [13, 14], which could activate monocytes to release myeloperoxidase and synthesize reactive oxygen species. Increased AOPPs levels were 
observed in IgAN patients [4, 13, 15]. Bilirubin, as a powerful endogenous antioxidant, plays an important role in oxidative stress. It can inhibit NADPH oxidase and further inflammatory reaction [9]. However, it is still do not know whether bilirubin has a role in IgAN progression.

Table 3: Analysis of factors associated with renal outcomes in model 2 (demographics + clinical indicators+ serum bilirubin).

\begin{tabular}{|c|c|c|c|c|c|c|}
\hline \multirow[t]{2}{*}{ Variables } & \multicolumn{3}{|c|}{ Before PSM } & \multicolumn{3}{|c|}{ After PSM } \\
\hline & HR & $95 \% \mathrm{CI}$ & $\mathrm{P}$ & HR & $95 \% \mathrm{CI}$ & $\mathrm{P}$ \\
\hline Male/female & 1.459 & $1.032-2.063$ & $0.033^{*}$ & 1.502 & $0.996-2.264$ & 0.052 \\
\hline Age (per year) & 0.978 & $0.963-0.993$ & $0.005^{*}$ & 0.985 & $0.967-1.004$ & 0.114 \\
\hline $\begin{array}{l}\text { Total bilirubin } \\
\text { (High/low) }\end{array}$ & 0.624 & $0.451-0.865$ & $0.005^{*}$ & 0.57 & $0.317-0.819$ & $0.002^{*}$ \\
\hline HTN(N/Y) & 0.981 & $0.696-1.383$ & 0.914 & 0.857 & $0.564-1.301$ & 0.469 \\
\hline ALT(IU/L) & 0.997 & $0.982-1.013$ & 0.73 & 0.999 & $0.981-1.017$ & 0.898 \\
\hline AST(IU/L) & 0.992 & $0.966-1.018$ & 0.545 & 0.994 & $0.963-1.027$ & 0.73 \\
\hline $\mathrm{TG}(\mathrm{mmol} / \mathrm{L})$ & 1.11 & $0.999-1.232$ & 0.052 & 1.006 & $0.866-1.168$ & 0.938 \\
\hline $\mathrm{TC}(\mathrm{mmol} / \mathrm{L})$ & 0.969 & $0.867-1.084$ & 0.583 & 1.113 & $0.976-1.268$ & 0.109 \\
\hline $\mathrm{UA}(\mathrm{umol} / \mathrm{L})$ & 1.003 & $1.002-1.005$ & $<0.001^{* *}$ & 1.004 & $1.002-1.006$ & $<0.001^{* *}$ \\
\hline URBC(/HP) & 0.998 & $0.997-1$ & $0.034^{*}$ & 0.998 & 0.996-1 & $0.038^{*}$ \\
\hline $\mathrm{LDH}(\mathrm{IU} / \mathrm{L})$ & 1.003 & $1.001-1.006$ & $0.017^{*}$ & 1.003 & $1-1.007$ & 0.057 \\
\hline $\begin{array}{l}\text { CKD stages } \\
\text { (stage1-3/4-5) }\end{array}$ & 0.162 & $0.111-0.237$ & $<0.001^{* *}$ & 0.179 & $0.114-0.28$ & $<0.001^{* *}$ \\
\hline NS(N/Y) & 0.793 & $0.465-1.352$ & 0.394 & 1.016 & $0.445-2.322$ & 0.97 \\
\hline Glucocorticoids (Y/N) & 0.861 & $0.633-1.172$ & 0.342 & 0.791 & $0.551-1.137$ & 0.206 \\
\hline Anemia (N/Y) & 0.586 & $0.4-0.858$ & $0.006^{*}$ & 0.621 & $0.374-1.029$ & 0.065 \\
\hline
\end{tabular}

Table 4: Analysis of factors associated with renal outcomes in model 3 (demographics+ clinical indicatorst pathological features + serum bilirubin).

\begin{tabular}{|c|c|c|c|c|c|c|}
\hline \multirow[t]{2}{*}{ Variables } & \multicolumn{3}{|c|}{ Before PSM } & \multicolumn{3}{|c|}{ After PSM } \\
\hline & $\begin{array}{l}\mathrm{HR} \\
\end{array}$ & $95 \%$ CI & $\mathrm{P}$ & HR & $95 \%$ CI & $\mathrm{P}$ \\
\hline Male/female & 1.215 & $0.863-1.71$ & 0.264 & 1.397 & $0.918-2.127$ & 0.118 \\
\hline Age (per year) & 0.98 & 0.964-0.996 & $0.015^{*}$ & 0.989 & $0.97-1.008$ & 0.251 \\
\hline $\begin{array}{l}\text { Total bilirubin } \\
\text { (High/low) }\end{array}$ & 0.651 & $0.467-0.906$ & $0.011^{*}$ & 0.568 & $0.394-0.819$ & $0.002^{*}$ \\
\hline HTN(N/Y) & 0.941 & $0.661-1.339$ & 0.735 & 0.919 & $0.597-1.414$ & 0.7 \\
\hline ALT(IU/L) & 0.995 & $0.979-1.011$ & 0.532 & 0.995 & $0.977-1.013$ & 0.568 \\
\hline AST(IU/L) & 0.999 & $0.972-1.026$ & 0.942 & 1.009 & $0.976-1.042$ & 0.607 \\
\hline $\mathrm{TG}(\mathrm{mmol} / \mathrm{L})$ & 1.097 & $0.987-1.219$ & 0.087 & 1.004 & $0.857-1.177$ & 0.956 \\
\hline $\mathrm{TC}(\mathrm{mmol} / \mathrm{L})$ & 0.995 & $0.883-1.122$ & 0.94 & 1.175 & $1.023-1.348$ & $0.022^{*}$ \\
\hline UA (umol/L) & 1.003 & $1.001-1.004$ & $0.001^{* *}$ & 1.003 & $1.001-1.005$ & $0.002^{*}$ \\
\hline URBC(/HP) & 0.999 & $0.997-1$ & 0.08 & 0.998 & $0.996-1$ & 0.071 \\
\hline LDH (umol/L) & 1.003 & $1-1.006$ & $0.041^{*}$ & 1.002 & $0.998-1.005$ & 0.319 \\
\hline $\begin{array}{l}\text { CKD stages } \\
\text { (stage1-3/4-5) }\end{array}$ & 0.241 & $0.161-0.36$ & $<0.001^{* *}$ & 0.272 & $0.17-0.436$ & $<0.001^{* *}$ \\
\hline $\mathrm{NS}(\mathrm{N} / \mathrm{Y})$ & 0.773 & $0.443-1.348$ & 0.364 & 1.134 & $0.483-2.664$ & 0.773 \\
\hline $\begin{array}{l}\text { Glucocorticoids } \\
(\mathrm{Y} / \mathrm{N})\end{array}$ & 0.974 & $0.713-1.331$ & 0.869 & 0.873 & $0.604-1.262$ & 0.47 \\
\hline Anemia(N/Y) & 0.686 & $0.412-1.141$ & 0.147 & 0.621 & $0.371-1.04$ & 0.07 \\
\hline M(M1/M0) & 1.13 & $0.706-1.81$ & 0.611 & 1.065 & $0.63-1.8$ & 0.813 \\
\hline E(E1/E0) & 1.196 & $0.647-2.212$ & 0.568 & 1.229 & $0.539-2.806$ & 0.624 \\
\hline $\mathrm{S}(\mathrm{S} 1 / \mathrm{S} 0)$ & 1.729 & $1.183-2.528$ & $0.005^{*}$ & 1.882 & $1.184-2.989$ & $0.007^{*}$ \\
\hline $\mathrm{T}(\mathrm{T} 1-2 / \mathrm{T} 0)$ & 2.681 & $1.882-3.819$ & $<0.001^{* *}$ & 2.582 & $1.702-3.916$ & $<0.001^{* *}$ \\
\hline $\mathrm{C}(\mathrm{C} 1-2 / \mathrm{C} 0)$ & 0.992 & $0.695-1.417$ & 0.965 & 0.873 & $0.555-1.372$ & 0.555 \\
\hline
\end{tabular}

Notes: NS nephrotic syndrome, ALT alanine aminotransferase, AST aspartate aminotransferase, UA uric acid, TG triglycerides, TC total cholesterol, URBC number of erythrocytes in urine at high magnification, LDH lactate dehydrogenase, HTN hypertension, M mesangial hypercellularity, E endocapillary hypercellularity, $\mathrm{S}$ segmental glomerulosclerosis or adhesion, $\mathrm{T}$ tubular atrophy/interstitial fibrosis,

$\mathrm{C}$ crescents, N: no, Y: yes.

*: $\mathrm{p}<0.05,{ }^{* *}: \mathrm{p} \leq 0.001$
In this study, in a cohort of 1492 patients with biopsy-proven IgAN, it was observed that a higher serum bilirubin level was beneficial to the outcome of IgAN. In the current study, it was found that the long-term renal outcome of IgAN patients with higher serum bilirubin level were better than those with lower serum bilirubin level. In the unmatched cohort, $16.2 \%(122 / 753)$ low-bilirubin group patients and $9.1 \%(67 / 739)$ high-bilirubin group patients progressed to the renal endpoints, and the KaplanMeier analysis revealed significant difference $(p<0.001)$. The result was consistent in the matched cohort $(\mathrm{p}=0.039)$, including $77(14.6 \%)$ patients in low bilirubin group and $57(10.1 \%)$ patients in high bilirubin group reached the endpoints.

In order to simulate randomization and improve balance on baseline variables, propensity score matching analysis was carried out. Multivariate Cox regression analysis was performed with several models consisted of demographics, clinical, pathological indexes and serum bilirubin level, which could contain more confounders to reduce selective bias. The results showed that serum bilirubin level was an independent risk factor in every model in both unmatched and matched cohort. Based on these results, serum bilirubin level could be recognized as a protective factor for long term prognosis of patients with IgAN. Previously, it was reported that serum bilirubin level was associated with the progression of IgAN [16, 17]. However, previous studies did not analyze the pathological changes, moreover, selective bias were not adjusted by PSM. Therefore, the results of this study are more reliable than previous studies.

Many studies focused on the relationship between serum bilirubin levels and some oxidative stress diseases including cardiovascular disease, diabetes, obesity [7-9]. The antioxidative characteristics of bilirubin might protect not only against the progression of CKD but also against loss of residual kidney function (RKF) in PD patients [18]. Elevated bilirubin concentrations were consistently associated with reduced vascular resistance [19, 20], improved eGFR [21], renal tubular function, and slowing of the progression of kidney damage [22]. In the animal experiments, the bilirubin-treated mice showed less fibrosis in the unilateral ureteral obstruction (UUO) model and bilirubin treatment decreased fibronectin expression in tubular epithelial cells [23]. Besides, it is reported that bilirubin interferes with vascular cell adhesion molecule1 (VCAM-1) and intercellular adhesion molecule1 (ICAM-1) to regulate atherosclerotic lesion formation and vascular inflammation both in animal and human models [9, 24]. Furthermore, bilirubin participates in immune response at several levels including 
modulation of regulatory $\mathrm{T}$ cells and $\mathrm{T}$ helper type 17 (Th17) cells, inhibition of the Toll-like receptor 4/nuclear factor kappaB signaling pathway, down-regulation of NLRP3 inflammasome [8, 24-26], which has been proved to be very important for IgA nephropathy in a previous study by our team [27].

However, there were still some limitations in our study. First, this was a single center study in China, the patients were mainly Han, Tibetan and Yi Chinese living in the southwest region of China, so it was not known whether our results had distinctive demographic or ethic characters. Second, though we adjusted for potential confounders which were likely to affect the association between serum bilirubin levels and kidney outcomes of IgAN patients, the effect of unadjusted factors such as serum ferritin, haptoglobin and the activity of the hemeoxygenase- 1 (HO-1), could not be eliminated in current study. Third, the sample size was limited which may not avoid all bias.

\section{Conclusion}

In conclusion, serum bilirubin level may be negatively associated with the progression of IgAN, which could be applied as a novel predictor in clinic. Future multicenter and perspective studies are needed to confirm these findings and to investigate the underlying mechanisms.

\section{Acknowledgements}

The authors thank all of the participants and attending physicians for their contributions.

\section{Availability of data and materials}

The datasets generated and/or analyzed during the current study are not publicly available due to privacy policy but are available from the corresponding author on reasonable request.

\section{Consent for publication}

Additional informed consent was obtained from all individual participants for whom identifying information is included in this article.

\section{Ethics approval and consent to participate}

This research was approved by the ethical committee of West China Hospital of Sichuan University.

\section{Funding}

This study was supported by grants from the project of the National Natural Science Foundation of China (No.8197031497) and Sichuan Province Science Foundation (No.18ZDYF2305).

\section{Author Contributions}

Conceptualization: ZJ, JXT, WQ. Data curation: ZJ, SQW, LQD, XH. Funding acquisition: YT, WQ. Data analysis and interpretation: ZJ, JXT. Manuscript writing: All authors. All authors read and approved the final manuscript.

\section{Competing Interests}

The authors have declared that no competing interest exists.

\section{References}

1. Rodrigues JC, Haas M, Reich HN. IgA Nephropathy. Clin J Am Soc Nephrol. 2017; 12: 677-86.

2. Wang Y, Tian J, Guo H, Mi Y, Zhang R, Li R. Intermedin ameliorates IgA nephropathy by inhibition of oxidative stress and inflammation. Clin Exp Med. 2016; 16: 183-92.

3. Apeland T, Mansoor MA, Furriol J, Ushakova A, Jonsson G, Stangeland KW, et al. Circulating inflammation-related factors are correlated with systemic redox status in IgA nephropathy; a case-control study. Free Radic Biol Med. 2020; $155: 10-8$

4. Tariq A, Mansoor MA, Marti HP, Jonsson G, Slettan A, Weeraman P, et al. Systemic redox biomarkers and their relationship to prognostic risk markers in autosomal dominant polycystic kidney disease and IgA nephropathy. Clin Biochem. 2018; 56: 33-40

5. Kobori H, Katsurada A, Ozawa Y, Satou R, Miyata K, Hase N, et al. Enhanced intrarenal oxidative stress and angiotensinogen in $\operatorname{IgA}$ nephropathy patients. Biochem Biophys Res Commun. 2007; 358: 156-63.

6. Soares MP, Bach FH. Heme oxygenase-1: from biology to therapeutic potential. Trends Mol Med. 2009; 15: 50-8.

7. Miao Liu , Jianhua Wang, He Y. The U-Shaped Association between Bilirubin and Diabetic Retinopathy Risk: A Five-Year Cohort Based on 5323 Male Diabetic Patients. Journal of Diabetes Research. 2018; Volume 2018, Article ID 4603087, : 7 pages.

8. Sundararaghavan VL, Binepal S, Stec DE, Sindhwani P, Hinds TD, Jr. Bilirubin, a new therapeutic for kidney transplant? Transplant Rev (Orlando). 2018; 32: 234-40

9. Vitek L. The role of bilirubin in diabetes, metabolic syndrome, and cardiovascular diseases. Front Pharmacol. 2012; 3: 55

10. Trimarchi H, Barratt J, Cattran DC, Cook HT, Coppo R, Haas M, et al. Oxford Classification of IgA nephropathy 2016: an update from the IgA Nephropathy Classification Working Group. Kidney Int. 2017; 91: 1014-21.

11. Zhu C, Mertens PR. IgA nephropathy and oxidative stress: news on clinically evaluated biomarkers hits the stage. Int Urol Nephrol. 2012; 44: 1277-80.

12. Coppo R, Camilla R, Amore A, Peruzzi L. Oxidative stress in IgA nephropathy. Nephron Clin Pract. 2010; 116: c196-8, discussion c9.

13. Duni A, Liakopoulos V, Roumeliotis S, Peschos D, Dounousi E Oxidative Stress in the Pathogenesis and Evolution of Chronic Kidney Disease: Untangling Ariadne's Thread. Int J Mol Sci. 2019; 20.

14. Poulianiti KP, Kaltsatou A, Mitrou GI, Jamurtas AZ, Koutedakis Y, Maridaki $\mathrm{M}$, et al. Systemic Redox Imbalance in Chronic Kidney Disease: A Systematic Review. Oxid Med Cell Longev. 2016; 2016: 8598253.

15. Wang J, Liang M, Xu J, Cao W, Wang GB, Zhou ZM, et al. Renal expression of advanced oxidative protein products predicts progression of renal fibrosis in patients with IgA nephropathy. Lab Invest. 2014; 94: 966-77.

16. Chin HJ, Cho HJ, Lee TW, Na KY, Oh KH, Joo KW, et al. The mildly elevated serum bilirubin level is negatively associated with the incidence of end stage renal disease in patients with IgA nephropathy. J Korean Med Sci. 2009; 24 Suppl: S22-9.

17. Tanaka S, Ninomiya T, Masutani K, Nagata M, Tsuchimoto A, Tsuruya K, et al. Prognostic impact of serum bilirubin level on long-term renal survival in IgA nephropathy. Clin Exp Nephrol. 2015; 19: 1062-70.

18. Tsujikawa H, Tanaka S, Hara M, Kawai Y, Matsukuma Y, Torisu K, et al. Association of Lower Serum Bilirubin With Loss of Residual Kidney Function in Peritoneal Dialysis Patients. Ther Apher Dial. 2020; 24: 202-7.

19. Bhuiyan AR, Srinivasan SR, Chen W, Sultana A, Berenson GS. Association of serum bilirubin with pulsatile arterial function in asymptomatic young adults: the Bogalusa Heart Study. Metabolism. 2008; 57: 612-6.

20. Fukui M, Tanaka M, Shiraishi E, Harusato I, Hosoda H, Asano M, et al. Relationship between serum bilirubin and albuminuria in patients with type 2 diabetes. Kidney Int. 2008; 74: 1197-201.

21. Shin HS, Jung YS, Rim H. Relationship of serum bilirubin concentration to kidney function and 24-hour urine protein in Korean adults. BMC Nephrol. 2011; 12: 29 .

22. Adin CA, Croker BP, Agarwal A. Protective effects of exogenous bilirubin on ischemia-reperfusion injury in the isolated, perfused rat kidney. Am J Physiol Renal Physiol. 2005; 288: F778-84. 
23. Park S, Kim DH, Hwang JH, Kim YC, Kim JH, Lim CS, et al. Elevated bilirubin levels are associated with a better renal prognosis and ameliorate kidney fibrosis. PLoS One. 2017; 12: e0172434.

24. Vogel ME, Zucker SD. Bilirubin acts as an endogenous regulator of inflammation by disrupting adhesion molecule-mediated leukocyte migration. Inflamm Cell Signal. 2016; 3.

25. Tsai MT, Tarng DC. Beyond a Measure of Liver Function-Bilirubin Acts as a Potential Cardiovascular Protector in Chronic Kidney Disease Patients. Int J Mol Sci. 2018; 20.

26. Liu Y, Li P, Lu J, Xiong W, Oger J, Tetzlaff W, et al. Bilirubin possesses powerful immunomodulatory activity and suppresses experimental autoimmune encephalomyelitis. J Immunol. 2008; 181: 1887-97.

27. Peng $\mathrm{W}$, Pei GQ, Tang $\mathrm{Y}$, Tan $\mathrm{L}$, Qin $\mathrm{W}$. IgA1 deposition may induce NLRP3 expression and macrophage transdifferentiation of podocyte in IgA nephropathy. J Transl Med. 2019; 17: 406. 